treated were checked only among their lifesaver colleagues. Since 2002, the education system of Japanese lifesavers has been reconstructed with a focus on medical control. Textbooks, that include CPR were revised in conjunction with the 2000 Guidelines, and the instructors were gathered and lectured about the changes and their scientific backgrounds. All lifesavers receive CPR Training Courses and learn how to use an automated external defibrillator (AED). Regional clubs that encountered a resuscitation case on the beach must report the details to the Medical Committee of the JLA. The Medical Committee of the JLA was informed about the prognosis of the patient transferred to hospital. Now ,the lifesavers are able to attend and see how to manage the drowning patient in some emergency centers, the final ring of the chain of survival. Conclusions: Lifesavers are the first responders for drowning patients and are an important part of improving the quality of the prehospital care especially for drowning victims.

Keywords: drowning; education; Japan; lifesavers; prehospital care Prehosp Disast Med 2005;20(2):s89-s90

Recommended Modifications and Applications of the Hospital Emergency Incident Command System J. Arnold; ${ }^{1}$ L. Dembry,; M. Tsai, ${ }^{2}$ U. Rodolpu, ${ }^{3}$ V. Parwani; ${ }^{1}$ J. Paturas; ${ }^{1}$ C. Cannon,; ${ }^{1}$ S. Selig ${ }^{1}$

1. Yale-New Haven Center for Emergency Preparedness and Disaster Response, USA

2. Department of Emergency Medicine, National Cheng Kung University Hospital, Taiwan

3. Department of Emergency Medicine, Alsancak State Hospital, Izmir, Turkey

Introduction: Since the inception of the Hospital Emergency Incident Command System (HEICS) in 1991, several events have transformed the requirements of hospital emergency management, including the 1995 Tokyo Subway sarin attack, the 2001 United States anthrax letter attacks, and the 2003 severe acute respiratory syndrome (SARS) outbreaks in eastern Asia and Toronto, Canada. Several modifications and new applications of the HEICS are suggested to match the needs of hospital emergency management today.

Methods: Recommendations were developed based on practical experiences with implementing the HEICS in acute-care hospitals and applying the HEICS to hospital emergency management in actual emergencies in Taiwan, Turkey, and the United States.

Results: It is recommended that the HEICS add: (1) an Incident Consultant in the Administration Section to provide expert advice directly to the Incident Commander in chemical, biological, radiation, and nuclear (CBRN) emergencies as needed; (2) new unit leaders in the Operations Section to coordinate the management of contaminated or infectious patients in CBRN emergencies; (3) new unit leaders in the Operations Section to coordinate mental health support to patients, guests, and healthcare workers in terrorism-related emergencies or events that produce significant mental health needs; (4) a new Decedent/Expectant Unit Leader in the Operations Section to coordinate the management of both types of patients together; and (5) a new Information Technology Unit Leader in the Logistics
Section to coordinate the management of information technology and systems. Also, new uses of the HEICS are recommended, including the adoption of the HEICS as the conceptual framework for organizing all phases of hospital emergency management and the application of the HEICS not only to healthcare facilities, but to healthcare systems as well. Finally, three levels of healthcare worker competencies in HEICS are suggested: (1) basic understanding of the HEICS for all hospital healthcare workers; (2) advanced understanding and proficiency in the HEICS for hospital healthcare workers likely to assume leadership roles in hospital emergency response; and (3) special proficiency in constituting the HEICS ad hoc from existing personnel for healthcare workers likely to work in resourcedeficient settings.

Conclusion: The HEICS should be viewed as a work in progress that will mature as additional challenges arise and hospitals gain further experience with its use.

Keywords: health care; hospital emergency incident command system (HEICS); recommendations

Prehosp Disast Med 2005;20(2):s90

\section{Estimated Vaccination of Population and Number of Clinics for Area Populations}

C. Doyle; A. Maber; J. Atas

Region 2S Medical Biodefense Network, USA

Part of the healthcare community response to an infectious disease emergency or disaster will be the ability to immunize a large population in a timely manner to prevent and interrupt the spread of disease. Vaccination clinics must be set up in convenient locations and in places that will not interfere with or interrupt the timely care of the sick at hospitals and emergency care centers. Recruitment and education of healthcare workers, as well as the establishment of clinic locations and hours of operation for the vaccination of vulnerable populations during the window of opportunity must be planned in advance.

A simulation vaccination clinic for smallpox was constructed to estimate the required time for vaccination of 1,000 patients including registration, orientation, screening, education, vaccination, mental health counseling, if needed, special needs access, and actual simulation of all components of an actual clinic. Plans for access, ingress and egress, and floor plans of the stations of the clinic were drafted in advance. Plans also were drafted to recruit screened and credentialed healthcare workers as volunteers.

These volunteer healthcare workers and volunteer patients were recruited to simulate all of the steps in an actual mass vaccination clinic. The simulation was timed from the first registration to the last discharge of patient volunteers to get an estimate of the timing required to vaccinate 1,000 patients. The number of clinics needed for a large population and the hours of operation required to vaccinate that population in a four-day window of exposure to a smallpox outbreak was estimated. The evaluation and vaccination process could be generalized to other infectious disease vaccination clinics.

The amount of supplies estimated for the 1,000 patients fully were utilized except for actual needles. Universal pre- 
cautions for infection such as changing gloves were simulated. The simulated total time to vaccinate 1,000 patients was just under four hours. Ambulance standby was arranged in case of actual illness of volunteers and in case screening uncovered any illness that would require immediate access to medical care. Security for clinic volunteers, healthcare workers, and vaccines also was simulated. Security was asked to screen for press credentials and direct all media to the designated public information officer (PIO) and to limit access to and protect the medical privacy of vaccine recipients. There was actual coverage of the simulation by local media. With these results regarding the required number of healthcare workers and required time to vaccinate 1,000 patients, numbers can be estimated for any size population depending on clinic operation hours of 12,16 , or 24 hours of operation. The number of healthcare workers required can be estimated depending on 8 or 12hour shifts.

Keywords: clinic; healthcare workers; population; simulation; vaccination

Prehosp Disast Med 2005;20(2):s90-s91

\section{Systematic Review of the Decontamination of Chemically Contaminated Casualties \\ I.W.F. Crawford; K.C. Mackway-Jones \\ Department of Emergency Medicine, Manchester Royal, United Kingdom}

Introduction: In the event of a chemical incident, whether the consequence of an accidental or deliberate release of toxic industrial chemicals or chemical warfare (CW) agents, there is a requirement for first responders to decontaminate potentially contaminated casualties. The purpose of the decontamination process is to physically remove, neutralize or destroy, or reduce to an acceptable level any chemical contaminant present, thereby reducing harm to the casualty and preventing secondary contamination of the first responders.

Objectives: The aim of this study was to determine the most effective approach to the decontamination of chemically contaminated casualties, specifically the need for and timing of decontamination and the effectiveness of: (1) clothing removal as the initial step in the decontamination process; (2) different decontaminants; and (3) different decontamination methods.

Methods: A number of specific, three-part questions were compiled to address the aims of this systematic review. The resources accessed in an effort to identify the literature available in the public domain were: (1) The Cochrane Library; (2) MEDLINE; (3) EMBASE; (4) CINAHL; (5) Science Direct; (6) ISI Web of Science; (7) ISI Proceedings; and (8) The Batelle Memorial Institute Mass-Casualty Decontamination Database. The resources accessed to identify the (potentially) classified literature not available in the public domain and the countries in which they exist were:

1. United Kingdom-Defence Science and Technology Laboratories (DSTL) Knowledge Services;

2. United States-Department of Defense (DoD) Chemical Biological Information Analysis Center (CBIAC);
3. Canada-Defence Research \& Development Canada (DRDC) Defence Research Reports Database; and

4. Australia-Defence Science and Technology Organisation (DSTO) Research Library.

Commercial manufacturers also were contacted. Studies were selected for inclusion based upon their relevance to the specific three-part questions. The studies could be published or unpublished scientific papers or technical reports.

Two reviewers independently selected the studies for inclusion and extracted relevant data. These data have been abstracted into evidence tables and appropriate conclusions have been drawn in the form of a series of clinical bottomlines for each of the specific three-part questions.

Results: The results of the completed process will be presented. Conclusions: A systematic review has determined the most effective approach to the decontamination of chemically contaminated casualties. The outcomes can be used to formulate best practice guidelines and advise first responders on the efficacy of the processes they already have in place and any changes that might be required for improvement.

Additionally, areas where further research is required have been identified.

Keywords: assessment; chemically contaminated casualties; decontamination; review

Prehosp Disast Med 2005;20(2):s91

Destruction of Conventional and Chemical Weapons from World War I (1914-1918), Ieper, BelgiumExample of Long-term Problems after War Situations $K$. Vandevelde

Belgium

Six years ago, Belgian military authorities developed a Destruction Unit for ammunition remaining from World War I. (WWI) in the area of Ieper, where several battles occurred. After WWI, thousands of tons of ammunition were dumped in the North Sea, at Zeebrugge, one kilometer from the coast. There are no available solutions for this problem in Zeebrugge. After the Oslo convention, dumping in the sea was forbidden. Historical stockpiles from WWI had to be destroyed. Between 1980-1996, 12,000 tons of ammunition were placed in Poelkappelle. Each year, 2,000 bombs are found, mostly by farmers. In April 2004,70 tons of ammunition were discovered in one place. WWI was the first time chemical weapons were used on a large-scale basis.

The Explosion Services of the army can be called after finding projectiles. In the Destruction Unit, ammunition is separated into high explosive and toxic chemical (10\%) weapons. A special procedure with nuclear, biological, chemical (NBC) clothing, RX apparatus, and spectrometrie is now available routinely in the Unit. There is an external and an internal disaster plan in the Destruction Unit.

One problem in case of accidents is the lack of knowledge by the medical rescue teams about the toxic effects and the treatment of war gasses (mustard gas, fosgeen, clark, etc.). Obligatory military service, the most important source of information about NBC problems during doctor and nurse education, no longer exists in Belgium. 